\title{
Decreased Angiogenic And Increased Apoptotic Activities Of Bone Microvascular Endothelial Cells In Patients With Glucocorticoid-Induced Osteonecrosis Of The Femoral Head
}

Huachen $\mathrm{Yu}$

Wenzhou Medical University https://orcid.org/0000-0001-7189-058X

Pei Liu

PLA Army General Hospital

Wei Zuo

China-Japan Friendship Hospital

Xiaowei Sun

Peking Union Medical College

Hongzhi Liu

Beijing University of Chinese Medicine

Feifan Lu

Peking University

Wanshou Guo

China-Japan Friendship Hospital

Qidong Zhang ( $\nabla$ zhangqidong1982@sina.com )

China-Japan Friendship Hospital

Research article

Keywords: Bone microvascular endothelial cells, angiogenesis, apoptosis, osteonecrosis of the femoral head, glucocorticoids

Posted Date: January 3rd, 2020

DOI: https://doi.org/10.21203/rs.2.20055/v1

License: (9) (1) This work is licensed under a Creative Commons Attribution 4.0 International License.

Read Full License 
Version of Record: A version of this preprint was published at BMC Musculoskeletal Disorders on April 29th, 2020. See the published version at https://doi.org/10.1186/s12891-020-03225-1. 


\section{Abstract}

Background: Glucocorticoid-induced osteonecrosis of the femoral head (ONFH) is closely associated with the dysfunction of the bone microvascular endothelial cells (BMECs). The present study investigated the angiogenic and apoptotic activity of the BMECs in glucocorticoid-induced ONFH.

Methods: This study enrolled a total of 12 patients, six of whom were assigned to the ONFH group whereas the other six served as the control group. The ONFH group was composed of patients with glucocorticoid-induced ONFH while the control group had femoral neck fractures. BMECs were isolated from the subchondral region of the femoral head. Cell proliferation, cell viability, tube formation assay, Transwell assay, TUNEL assay, and Western blot analysis were performed.

Results: No significant differences were noticed in BMECs proliferation between the two groups. However, compared to the control, cell viability, tube formation, and migration of BMECs were significantly decreased and the number of TUNEL positive cells was markedly increased in the ONFH group. In the ONFH group, it was also noted that the amount of Bax and cleaved-caspase3 was elevated while that of Bcl-2 was reduced.

Conclusion: The findings of our study revealed that BMECs obtained from the glucocorticoid-induced ONFH patients had decreased angiogenic and increased apoptotic activities, which could explain the pathogenesis and progression of glucocorticoid-induced ONFH.

\section{Background}

Glucocorticoids are often used to treat diseases such as severe acute respiratory syndrome, organ transplantation, acute lymphoblastic leukemia, multiple myeloma, rheumatoid arthritis, and systemic lupus erythematosus [1-5]. Prolonged usage of glucocorticoids is a proven cause of ONFH. The ONFH is a disabling condition and often occurs in individuals between 20 and 50 years old [6]. Approximately $65 \%$ of ONFH patients eventually require total hip replacements, resulting in a substantial economic and medical burden to the patient and society [7]. Although the relationship between glucocorticoid administration and the development of glucocorticoid-induced ONFH is a well-known phenomenon, the mechanism of its pathogenesis remains obscure [8].

Recent reports have indicated that the femoral head microcirculation obstacle caused by a dysfunction of the bone microvascular endothelial cells (BMECs) could be significant in the development of glucocorticoid-induced ONFH[9-11].BMECs line the sinusoids and inner layer of blood vessels and play a crucial role in vascular homeostasis and angiogenesis [12]. Also, the regulation of apoptosis during angiogenesis occurs in the BMECs [13]. It has been reported that the functioning of angiogenesis and blood vessel integrity is negatively related to the level of apoptosis [14]. Besides, several studies have reported that the dysfunction of regional endothelial cells due to continuous exposure to glucocorticoids can induce cell apoptosis and inhibit angiogenesis [15-16]. However, there are currently no reports on 
whether the angiogenic and apoptotic activity of BMECs is affected in glucocorticoid-induced ONFH patients.

This study postulated that the angiogenic and apoptotic activity of BMECs could be altered in patients with glucocorticoid-induced ONFH. This study tested the above hypothesis by investigating the angiogenic and apoptotic activities of BMECs isolated from patients with glucocorticoid-induced ONFH. A control group consisting of patients with femoral neck fractures was used.

\section{Methods}

\section{Patients}

The specimens were obtained from the Orthopedic Department of the hospital between March 2018 and November 2018. A total of 12 participants were enrolled, six of whom had glucocorticoid-induced ONFH. These six patients formed the ONFH group while the other six had femoral neck fractures, and these formed the control group. The inclusion criteria were (1) male or female patients between 40 and 70 years old; (2) diagnosis of glucocorticoid-induced ONFH or femoral neck fracture; (3) indications for total hip arthroplasty. The exclusion criteria included (1) alcohol-induced or trauma-induced ONFH and (2) preoperative diagnosis of either HIV, hepatitis B or C infections. The ONFH group comprised 3 women and 3 men (average age of $51.7 \pm 5.2$ years) at the time of surgery, while the control group comprised five women and one man (average age of $65.3 \pm 2.9$ years) at the time of surgery (Table 1 ). Femoral heads were removed during total hip arthroplasty, and a band saw was used to cut all the specimens into half at the coronary level. Half of the femoral heads were fixed in $10 \%$ formalin for examination while the remaining half was used for the isolation and culture of BMECs.

\section{Hematoxylin-eosin(HE) staining}

The collected tissues were fixed in $10 \%$ formalin after which they were decalcified in $10 \%$ EDTA solution for six weeks. The samples were subsequently dehydrated in a graded series of ethanol, embedded in paraffin, sliced into $5 \mu \mathrm{m}$ thick sections. After staining with $\mathrm{HE}$, a light microscope was used to examine the level of necrosis of the bone and marrow tissues. The existence of empty lacuna was used to indicate the level of osteonecrosis [17]. The average number of lacunae from three fields was calculated for each group.

\section{Isolation and culture of BMECs}

The BMECs of patients undergoing hip arthroplasty were obtained as described previously [18]. Briefly, cancellous bones were harvested from the subchondral region of the femoral head. The bone debris was digested with $0.2 \%$ type I collagenase and $0.25 \%$ Trypsin-EDTA for 5 minutes. The reaction was stopped with the addition of Dulbecco's modified Eagle's medium (DMEM, Gibco, USA). The cell lysates were filtered with 70- $\mu \mathrm{mol} / \mathrm{L}$ cell strainer and centrifugated at $1500 \mathrm{rpm}$ for $6 \mathrm{~min}$. The supernatant was removed and the cells were bathed in endothelial cell medium (ECM, ScienCell, USA) containing $5 \%$ fetal 
bovine serum (FBS), $5 \mathrm{ml}$ recombinant human VEGF and antibiotics in a $37^{\circ} \mathrm{C}$ humidified incubator with $5 \% \mathrm{CO}_{2}$. A fresh medium was added the next day, and at intervals of three days subsequently. The cells were passaged when they reached $90 \%$ confluence.

\section{Examination of cell proliferation and viability}

The proliferation of BMECs was assessed using the CCK-8 kit following the instructions on the kit. After BMCEs incubation in the 96 wells, $100 \mu \mathrm{L}$ of ECM and $10 \mu \mathrm{L}$ of CCK-8 solution were added to each well for a 2 -h incubation period. The OD of each well was determined using a microplate reader at a wavelength of $450 \mathrm{~nm}$.

The neutral red uptake assay was used to assess cell viability, as detailed before [19]. BMECs were added to 96 -well plate $\left(2 \times 10^{5}\right.$ cells/well) and allowed to adhere. Next, a fresh FBS-free medium was added and then the cells were treated with neutral red dye (in a fresh medium) for $2 \mathrm{~h}$. Subsequently, a neutral red lysis buffer was used to remove the neutral red stain, and the OD was assessed at $570 \mathrm{~nm}$.

\section{TUNEL assay}

Apoptosis of BMECs was measured with TUNEL staining using the In Situ Cell Death Detection Kit (Roche). Cultured BMECs were collected in a six-well plate, $12 \mathrm{~h}$ post preparation. The cells were fixed with freshly prepared $4 \%$ paraformaldehyde for $1 \mathrm{~h}$, incubated with $3 \% \mathrm{H}_{2} \mathrm{O}_{2}$ and $0.1 \%$ Triton X-100 for 10 min, and washed thrice with phosphate-buffered saline (PBS) during each step. Cells were subsequently stained with DAPI according to the In Situ Cell Death Detection Kit manual. Images were observed and captured using a fluorescence microscope (Olympus, Tokyo, Japan). The number of apoptotic cells was quantified by counting TUNEL-positive cells by three independent researchers.

\section{Tube formation assay}

A 96-well plate was coated with $100 \mu \mathrm{L}$ of Matrigel (BD, USA) and then allowed to solidify and polymerize at $37^{\circ} \mathrm{C}$ for $1 \mathrm{~h}$. The Matrigel was subsequently overlaid with $100 \mu \mathrm{L}$ of a suspension of BMECs $\left(2 \times 10^{5}\right.$ cells/well). Tube formation by BMECs was observed under a microscope after $6 \mathrm{~h}$ of incubation and quantified by NIH Image J.

\section{Transwell assay}

Corning transwell chambers (Corning, $8 \mu \mathrm{m}$, USA) were used for the transwell assay. For the migration assay, $5 \times 10^{5}$ BMECs suspended in $200 \mu \mathrm{L}$ serum-free medium were added in the upper chambers, and $500 \mu \mathrm{L}$ ECM containing FBS were added in the lower chamber. Following incubation for $12 \mathrm{~h}$, the upper chambers were washed with PBS, and the cells on the upper surface were wiped off using a cotton swab. Subsequently, cells on the bottom surface of the membrane were fixed in $4 \%$ paraformaldehyde and stained with $1 \%$ crystal violet. The number of migrated cells was assessed and counted under an optical microscope. 


\section{Western blot analysis}

Cellular total protein was extracted using RIPA lysis buffer and then quantified using the BCA kit (Beyotime, China). After that, proteins were denatured by heating at $95^{\circ} \mathrm{C}$ for $5 \mathrm{~min}$. A $30 \mu \mathrm{g}$ sample of proteins were resolved by $10 \%$ SDS-PAGE and transferred to a PVFD membrane. The membrane was bathed in fat-free milk to block non-specific binding for $1 \mathrm{~h}$ followed by incubating overnight at $4^{\circ} \mathrm{C}$ with primary antibodies against caspase-3 (Abcam, 1:1000), cleaved caspase-3 (Abcam, 1:1000), Bax (Abcam, 1:1000), Bcl-2 (Abcam,1:500), and $\beta$-actin (Abcam,1:3000). Subsequently, they were incubated with their corresponding secondary antibodies. The immunoblots were visualized with Electrochemiluminescence Plus Reagent (Invitrogen), and the expression was quantified with Image Lab 3.0 software.

\section{Statistical analysis}

All data were expressed as the mean \pm standard deviation (SD). Data analysis was done using one-way analysis of variance (ANOVA) and the Student's t-test using SPSS version 21.0 (SPSS Inc., Chicago, IL, USA). A value of $P<0.05$ was considered statistically significant.

\section{Results}

\section{Clinical data and histological analysis}

Representative femoral heads of the glucocorticoid-induced ONFH and the femoral neck fracture are indicated in Fig 1A and 1B, respectively. Femoral head necrosis and bone marrow edema occurred in the ONFH group.

HE staining results showed that the bone marrow structure was disordered. Bone marrow necrosis and empty bone lacunae were observed in the ONFH group, while the control group had thick bone trabeculae and normal osteocytes (Fig 1C-D). The proportion of empty bone lacunae was relatively higher in the ONFH group in comparison to the control group $(P<0.01)$, as shown in Fig $1 \mathrm{E}$

\section{Cell morphology, proliferation, and viability of BMECs}

The morphologies of the P2 BMECs in the ONFH and control groups are shown in Fig. 2A and B, respectively. Cell proliferation was evaluated 2, 4, and 6 days during the proliferation assay. No significant differences in cell proliferation were recorded between the two groups $(P>0.05 ; \mathrm{Fig}$. $2 \mathrm{C})$. With regards to cell viability, however, more neutral red-stained cells were observed in the control groups than the ONFH group $(\mathrm{P}<0.05$; Fig. 2D-F).

\section{Glucocorticoid decreased the angiogenic activity of BMECs in the ONFH group}

We used the tube formation and migration assays to compare the angiogenic activities of BMECs in the two groups. Whereas BMCEs in the control group formed elongated, vessel-like structures, the ONFH group BMECs formed incomplete or sparse tubular networks $(P<0.01$ Fig. $3 A-C)$. Migration capacity was 
remarkably decreased in the ONFH group relative to the control group $(P<0.01 \mathrm{Fig}$. 3D-F). Based on the results, this study concluded that BMECs in the ONFH group showed a significantly low angiogenic activity in comparison to the control group.

\section{Glucocorticoid increased apoptosis activity of BMECs in the ONFH group}

We first used the TUNEL assay to compare the apoptotic activities of the BMECs of the two groups. The TUNEL assay revealed that the apoptosis of BMECs was markedly higher in the ONFH group versus the control group ( $P<0.05$ Fig. 4A-C). Further attempts were made to assess the expression of proteins related to apoptosis such as Bcl-2, cleaved-caspase 3, caspase 3 and Bax. Our results showed that Bcl-2 was markedly decreased, while Bax was elevated in the ONFH group, relative to the control group. Moreover, it was noted that the expression levels of cleaved caspase 3 were notably elevated in the ONFH group $(P<0.05$ Fig. 4D-G).

\section{Discussion}

Glucocorticoid-induced ONFH is an intractable and complex orthopedic disease caused by long term use or overdose of glucocorticoid treatment [8]. Studies have shown that the development of glucocorticoidinduced ONFH is closely associated with the dysfunction of BMECs [10]. This study reveals for the first time that BMECs obtained from patients with glucocorticoid-induced ONFH have decreased angiogenic and increased apoptotic activities compared with BMECs from patients with femoral neck fractures.

Although many theories regarding glucocorticoid-induced ONFH have been put forward, the underlying mechanism remains unclear. The dysfunction of BMECs has been reported to cause femoral head microcirculation obstacle, which is key to the development of glucocorticoid-induced ONFH $[9,20]$. Yu et al. [21] reported that glucocorticoids profoundly affect the transcriptome of BMECs during the early stages of glucocorticoid-induced ONFH. According to Yue et al. [18], glucocorticoids can induce BMECs to express various cytokines, which contributes to the development of glucocorticoid-induced ONFH. In the present investigation, we used tube formation and migration assays to examine angiogenesis. The findings of this study indicate that the angiogenic activity of BMECs was profoundly impaired in the ONFH group in comparison with the control group. The reduced angiogenic activity of BMECs in ONFH patients may, therefore, reflect dysfunction of BMECs, and is thus a potential mechanism for glucocorticoid-induced ONFH. It could suggest that ONFH patients with the decreased angiogenic activity of BMECs frequently develop ONFH because of an impaired vascular repair and blood vessel growth.

In our study, patients in the control group, in which patients had femoral neck fractures, were significantly older than those in the ONFH group. The distinct features of the two conditions caused inevitable biases in the age of the two groups. Aging has been reported to be associated with endothelial dysfunction [2223]. For instance, the study by Yavuz et al. [24] reported that endothelial function declines with an increase in the age of healthy human subjects. In our study, the angiogenesis of BMECs in the ONFH group was remarkably decreased as relative to the control group, even though the ONFH patients were 
much younger. This finding suggests that glucocorticoids accelerate the dysfunction of BMECs in ONFH patients.

The mechanisms driving the dysfunction of BMECs in the ONFH group have, however, not been determined. One of the hypotheses postulates that it might be due to increased apoptosis of BMECs. Kerachian et al. [16] pointed out that apoptosis of BMECs could activate the thrombosis cascade, followed by ischemia and infarction. Also, Vogt et al. [20] showed that glucocorticoids could reduce angiogenesis by inducing the apoptosis of endothelial cells, and therefore injuring BMECs. In this study, the TUNEL-positive cells were only recorded in the ONFH group, suggesting that glucocorticoids induced apoptosis of BMECs in ONFH patients. It is known that caspase cascade plays an essential role in apoptosis and caspase- 3 is activated during apoptosis [25]. Overexpression of caspase-3 has previously reported in ONFH patients [26]. Disruption of mitochondrial functions due to oxidative stress factors stimulates the caspase pathway, triggering apoptosis [27]. Also, the stimulation of cells by apoptotic factors facilitates dissociation of the original Bax and Bcl-2, causing an increase in Bax and decrease in $\mathrm{Bcl}-2$ [28]. In this study, we demonstrated that Bcl-2 was markedly suppressed, while Bax and cleaved caspase 3 were increased in the ONFH group. These changes in expression levels suggest that the mitochondria participate in the apoptosis of BMECs in ONFH patients.

The following shortcoming should be underscored in this study. Firstly, the study was done in vitro and cannot, therefore, be replicated in the local environment of the femoral head, in the setting of glucocorticoid-induced ONFH. Consequently, the dysfunction of BMECs reported in this study may not hold for other etiologies of glucocorticoid-induced ONFH. Secondly, the sample size of our study was small and cannot, therefore, account for all patient comorbidities.

\section{Conclusion}

In summary, the findings of our study indicate that BMECs obtained from glucocorticoid-induced ONFH patients have decreased angiogenic and increased apoptotic activities, which, to our knowledge, has not been previously reported. The decreased angiogenic and increased apoptotic activities may be responsible for the pathogenesis and progression of glucocorticoid-induced ONFH. Protecting the function of BMECs could, therefore, offer an efficient therapeutic target for glucocorticoid-induced ONFH, which needs further exploration.

\section{Abbreviations}

ONFH: Osteonecrosis of the femoral head; BMECs: Bone microvascular endothelial cells; HE: Hematoxylin-eosin; DMEM : Dulbecco's modified Eagle's medium; ECM: Endothelial cell medium; FBS: Fetal bovine serum; PBS: Phosphate-buffered saline; SD:Standard deviation; one-way ANOVA: One-way analysis of variance.

\section{Declarations}




\section{Ethics approval and consent to participate}

This study was performed in conformity with the Helsinki declarations and all protocols were approved and monitored by the Institutional Review Board of the China-Japan Friendship Hospital (Ethical commission Number 2018-149-K106). All participants provided informed consent.

\section{Consent for publication}

Not applicable.

\section{Availability of data and materials}

The datasets used and/or analyzed during the current study are available from the corresponding author on reasonable request.

\section{Competing interests}

The authors declare that they have no competing interests.

\section{Funding}

This work was supported by by the National Natural Science Foundation of China (No. 81703896 and No.81673776), Beijing municipal science and technology commission (No. Z171100001017209), the Capital Health Research and Development of Special (No. 2016-2-4062) and Wenzhou Municipal Science and Technolgoy Burea (Y20180030) and Zhejiang Medical and Health Science and Technology Project (2020KY181). The funding body has not been involved in the design, data collection, analysis, interpretation or the writing of the manuscript.

\section{Authors' contributions}

$\mathrm{HCY}$ and PL carried out the main part of the studies and participated in manuscript preparation. WZ and XWS helped with the experiment. HZL and FFL participated in the statistical analysis.WSG and QDZ designed and instructed the conduct of the research. All authors have read and approved the final manuscript.

\section{Acknowledgements}

The authors thank the staff of the China-Japan Friendship Institute of Clinical Medicine, Beijing, China.

\section{References}

1. Tanaka Y, Yoshikawa N, Hattori S, Sasaki S, Ando T, Ikeda M, Honda M. Combination therapy with steroids and mizoribine in juvenile SLE: a randomized controlled trial. Pediatr Nephrol. 2010; 25(5):877-82. 
2. Hodson EM, Alexander SI. Evaluation and management of steroid-sensitive nephrotic syndrome. Curr Opin Pediatr. 2008; 20(2):145-50.

3. Chan MH, Chan PK, Griffith JF, Chan IH, Lit LC, Wong CK, Antonio GE, Liu EY, Hui DS, Suen MW et al. Steroid-induced osteonecrosis in severe acute respiratory syndrome: a retrospective analysis of biochemical markers of bone metabolism and corticosteroid therapy. Pathology. 2006; 38(3):229-35.

4. Marston SB, Gillingham K, Bailey RF, Cheng EY. Osteonecrosis of the femoral head after solid organ transplantation: a prospective study. J Bone Joint Surg Am. 2002; 84(12):2145-51.

5. Talamo G, Angtuaco E, Walker RC, Dong L, Miceli MH, Zangari M, Tricot G, Barlogie B, Anaissie E. Avascular necrosis of femoral and/or humeral heads in multiple myeloma: results of a prospective study of patients treated with dexamethasone-based regimens and high-dose chemotherapy. $J$ Clin Oncol. 2005; 23(22):5217-23.

6. Zalavras CG, Lieberman JR: Osteonecrosis of the femoral head: evaluation and treatment. J Am Acad Orthop Surg. 2014; 22(7):455-64.

7. Fukushima W, Fujioka M, Kubo T, Tamakoshi A, Nagai M, Hirota Y. Nationwide epidemiologic survey of idiopathic osteonecrosis of the femoral head. Clin Orthop Relat Res. 2010; 468(10):2715-24.

8. Weinstein RS. Glucocorticoid-induced osteonecrosis. Endocrine. 2012; 41(2):183-90.

9. Nishimura T, Matsumoto T, Nishino M, Tomita K. Histopathologic study of veins in steroid treated rabbits. Clin Orthop Relat Res. 1997;(334):37-42.

10. Kerachian MA, Seguin C, Harvey EJ. Glucocorticoids in osteonecrosis of the femoral head: a new understanding of the mechanisms of action. J Steroid Biochem Mol Biol. 2009; 114(3-5):121-28.

11. Seguin C, Kassis J, Busque L, Bestawros A, Theodoropoulos J, Alonso ML, Harvey EJ. Non-traumatic necrosis of bone (osteonecrosis) is associated with endothelial cell activation but not thrombophilia. Rheumatology (Oxford). 2008; 47(8):1151-55.

12 Kusumbe AP, Ramasamy SK, Adams RH. Coupling of angiogenesis and osteogenesis by a specific vessel subtype in bone. Nature. 2014; 507(7492):323-8.

13. Williams TA, Verhovez A, Milan A, Veglio F, Mulatero P. Protective effect of spironolactone on endothelial cell apoptosis. Endocrinology. 2006;147(5):2496-505.

14. O'Connell BJ, Genest J, Jr. High-density lipoproteins and endothelial function. Circulation. 2001; 104(16):1978-83.

15. Zhang Y, Yin J, Ding H, Zhang C, Gao YS. Vitamin K2 Ameliorates Damage of Blood Vessels by Glucocorticoid: a Potential Mechanism for Its Protective Effects in Glucocorticoid-induced Osteonecrosis of the Femoral Head in a Rat Model. Int J Biol Sci. 2016;12(7):776-85.

16. Kerachian MA, Harvey EJ, Cournoyer D, Chow TY, Seguin C. Avascular necrosis of the femoral head: vascular hypotheses. Endothelium. 2006; 13(4): 237-44.

17. Steffen RT, Athanasou NA, Gill HS, Murray DW. Avascular necrosis associated with fracture of the femoral neck after hip resurfacing: histological assessment of femoral bone from retrieval 
specimens. J Bone Joint Surg Br. 2010; 92(6):787-93.

18. Yue J, Wan F, Zhang Q, Wen P, Cheng L, Li P, Guo W. Effect of glucocorticoids on miRNA expression spectrum of rat femoral head microcirculation endothelial cells. Gene. 2018; 651:126-33.

19. Abud AP, Zych J, Reus TL, Kuligovski C, de Moraes E, Dallagiovanna B, de Aguiar AM.The use of human adipose-derived stem cells based cytotoxicity assay for acute toxicity test. Regul Toxicol Pharmacol.2015; 73(3):992-8.

20. Vogt CJ, Schmid-Schonbein GW. Microvascular endothelial cell death and rarefaction in the glucocorticoid-induced hypertensive rat. Microcirculation. 2001; 8(2):129-39.

21. Yu QS, Guo WS, Cheng LM, Lu YF, Shen JY, Li P. Glucocorticoids Significantly Influence the Transcriptome of Bone Microvascular Endothelial Cells of Human Femoral Head. Chin Med J (Engl). 2015; 128(14):1956-63.

22 Sepulveda C, Palomo I, Fuentes E. Mechanisms of endothelial dysfunction during aging:

Predisposition to thrombosis. Mech Ageing Dev. 2017; 164:91-9.

23 Mazzoccoli G, Fontana A, Grilli M, Dagostino MP, Copetti M, Pellegrini F, Vendemiale G. Idiopathic deep venous thrombosis and arterial endothelial dysfunction in the elderly. Age (Dordr). 2012;34(3):75160 .

24 Yavuz BB, Yavuz B, Sener DD, Cankurtaran M, Halil M, Ulger Z, Nazli N, Kabakci G, Aytemir K, Tokgozoglu $\mathrm{L}$ et al. Advanced age is associated with endothelial dysfunction in healthy elderly subjects. Gerontology. 2008; 54(3):153-6.

25 Porter AG, Janicke RU. Emerging roles of caspase-3 in apoptosis. Cell Death Differ. 1999; 6(2):99104.

26 Xu X, Wen H, Hu Y, Yu H, Zhang Y, Chen C, Pan X. STAT1-caspase 3 pathway in the apoptotic process associated with steroid-induced necrosis of the femoral head. J Mol Histol. 2014;45(4):473-85.

27 Murphy KM, Ranganathan V, Farnsworth ML, Kavallaris M, Lock RB. Bcl-2 inhibits Bax translocation from cytosol to mitochondria during drug-induced apoptosis of human tumor cells. Cell Death Differ. 2000; 7(1):102-11.

28 Hu WP, Yu HS, Sung PJ, Tsai FY, Shen YK, Chang LS, Wang JJ. DC-81-Indole conjugate agent induces mitochondria mediated apoptosis in human melanoma A375 cells. Chem Res Toxicol. 2007; 20(6):905-12.

\section{Table}

Table 1 Comparison of Patients in the ONFH and control group 


\begin{tabular}{lccc}
\hline Demographics & ONFH $(\mathrm{n}=6)$ & Femoral neck fracture $(\mathrm{n}=6)$ & $P$-value \\
\hline Males & 3 & 1 & 0.545 \\
Females & 3 & 5 & \\
\hline Mean ages $\square \mathrm{y} \square$ & $51.7 \pm 5.2$ & $65.3 \pm 2.9$ & $<0.01$ \\
\hline
\end{tabular}

Figures
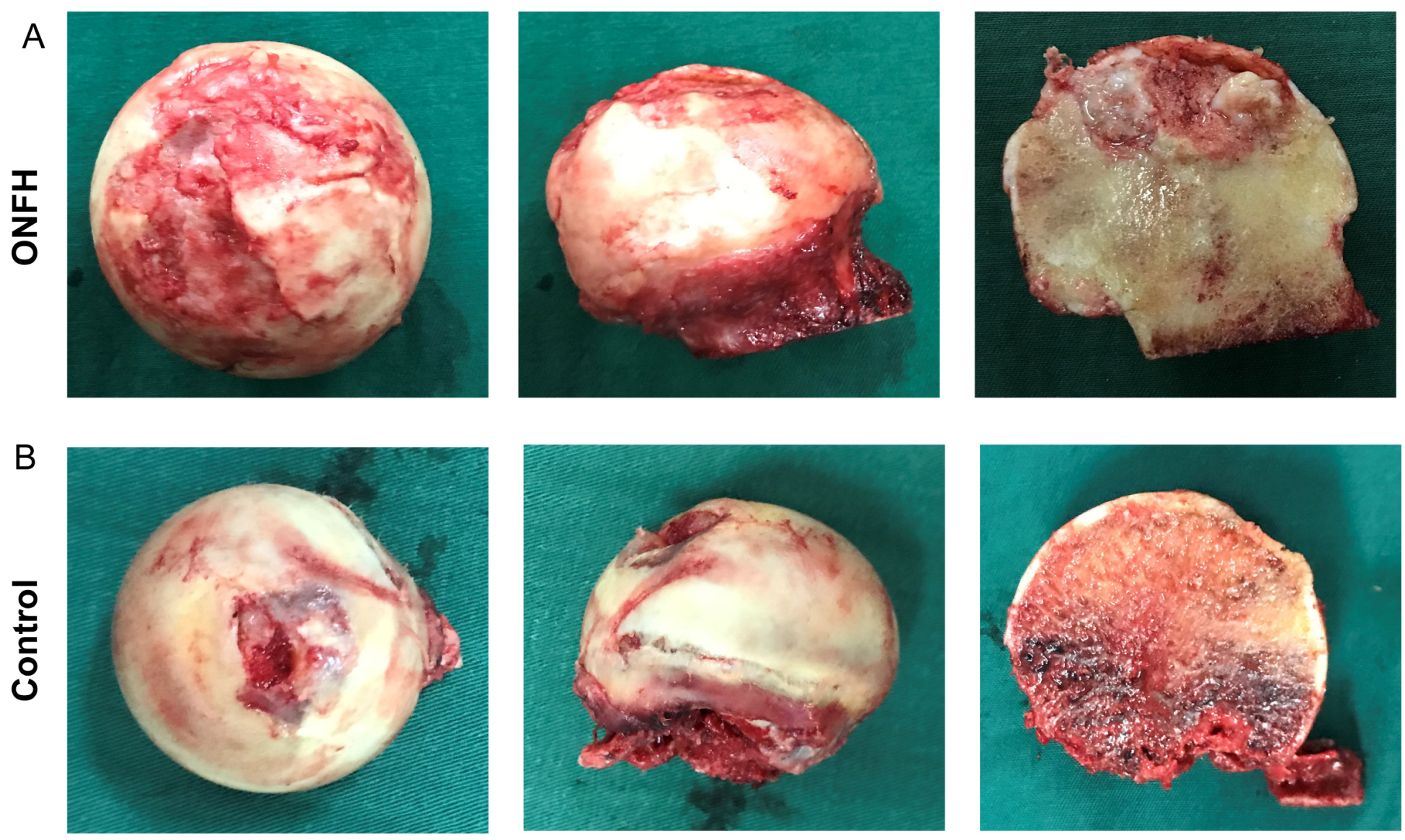

ONFH

\section{Control}
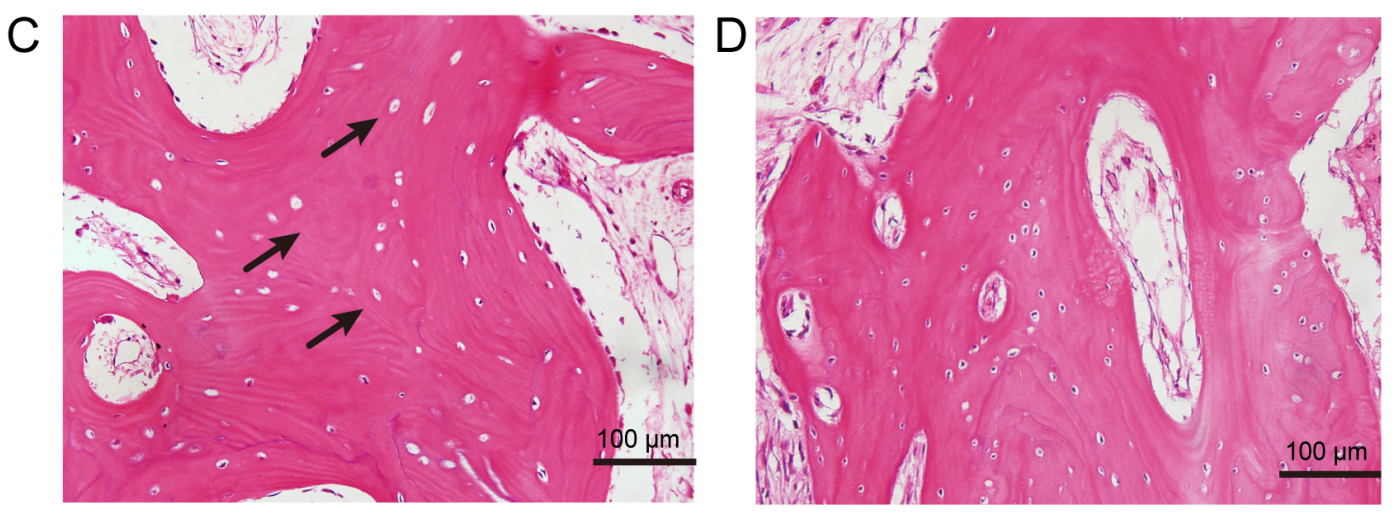

E

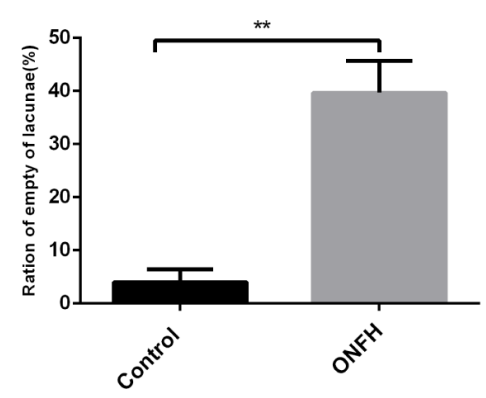

Figure 1 
Clinical data of patients. Representative images of femoral heads from the ONFH (A) and the control group (B). Histological analysis of femoral heads (C-E). HE staining showing a disordered bone marrow structure and bone marrow necrosis, and empty bone lacunae found in the ONFH group. There was no ONFH in the control group. Bars depict the ratio of empty lacunae. Black arrows indicate empty lacunae (** indicates $\mathrm{P}<0.01$ ).

ONFH

A

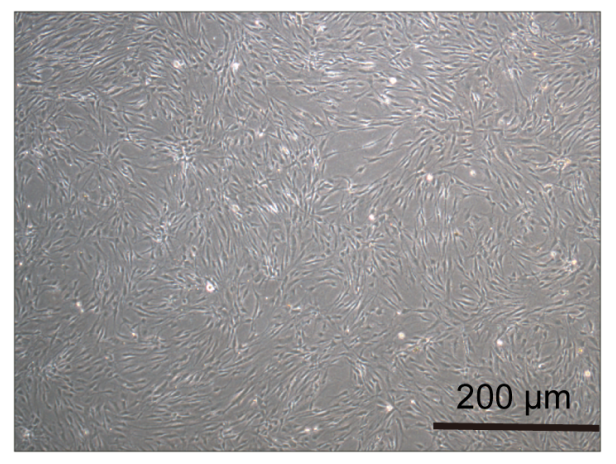

D

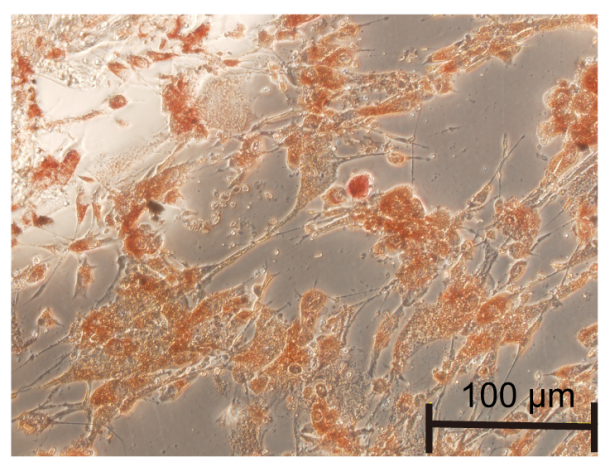

Control

B

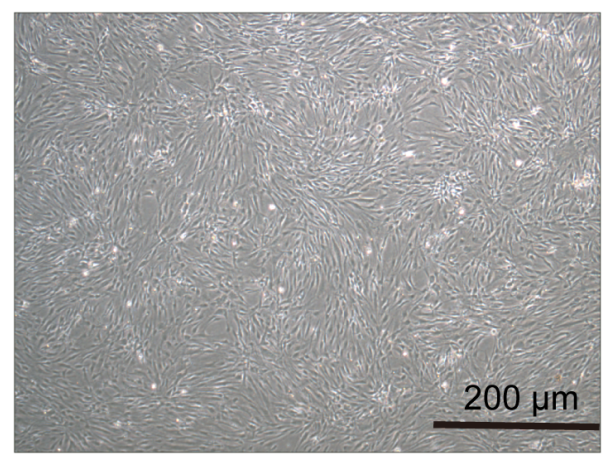

E

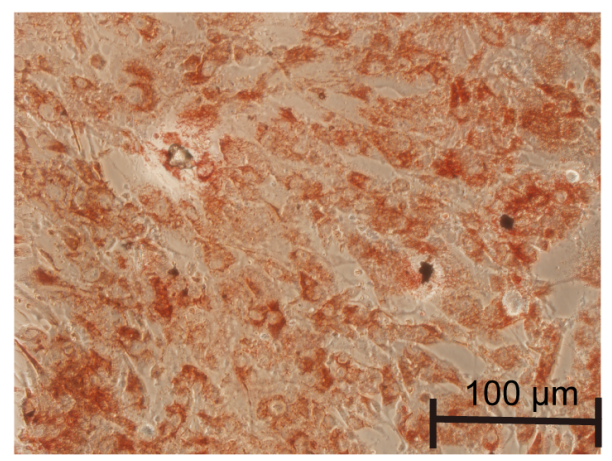

C

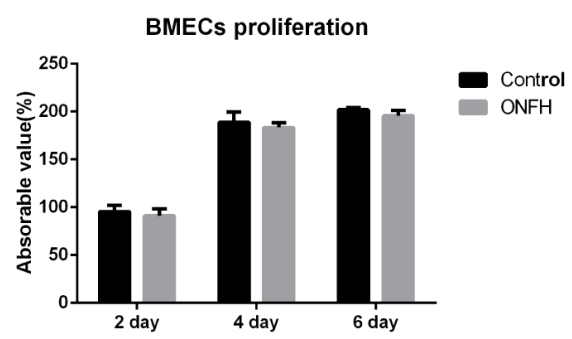

$\mathrm{F}$

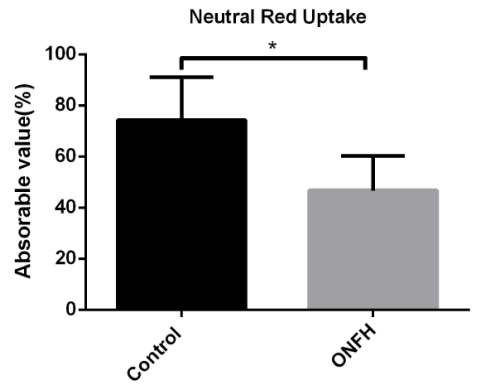

Figure 2

Comparison of morphology and growth potential of BMECs (at P2) from the group (A) and the control group (B). (C) Cell proliferation rates of BMECs in the ONFH and control groups. (D-F) Neutral red uptake assay showed fewer neutral red-stained cells in the ONFH group, but more red-stained cells in the control group ( $*$ indicates $\mathrm{P}<0.05$ ). 
ONFH

A

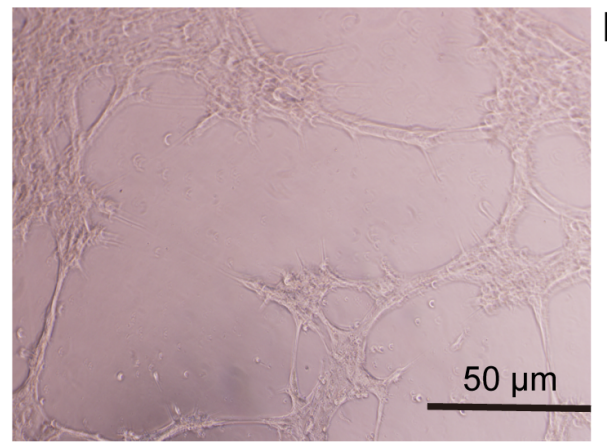

$\mathrm{D}$

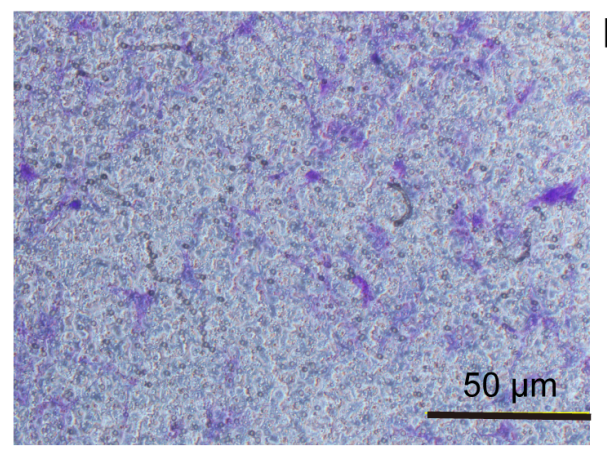

Control

B
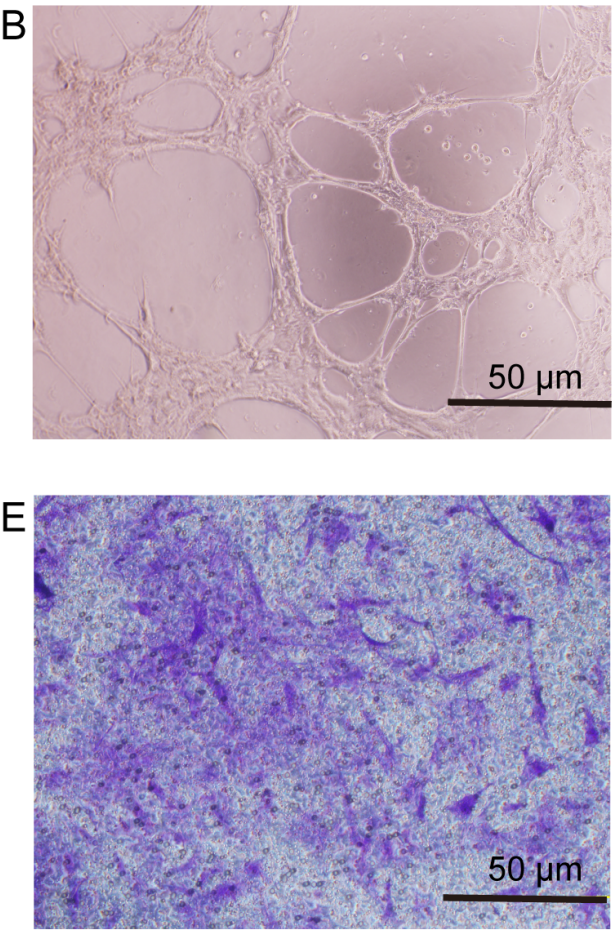

C

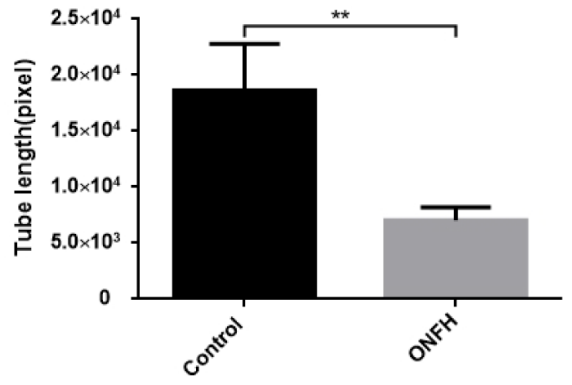

$\mathrm{F}$

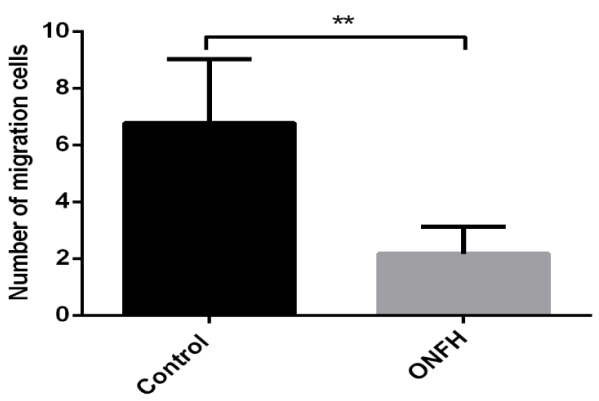

\section{Figure 3}

Angiogenic activity of BMECs. (A-B)Representative images of the tube formation assay from the ONFH group and the control group. BMECs from the ONFH group did not form tube-like structures on Matrigel, but control group BMECs successfully tube-like structure. (C) Quantification analysis of total tube length of BMECs ( $\star \star$ indicates $P<0.01$ ). (D-E) Typical results of the transwell assay of the ONFH group and the control group at $6 \mathrm{~h}$ are shown. BMECs from the ONFH group had a lower proportion of spreading cells (violet-stained) compared to the control group. (F) Quantification analysis of number migration cells ( * indicates $\mathrm{P}<0.01)$. 

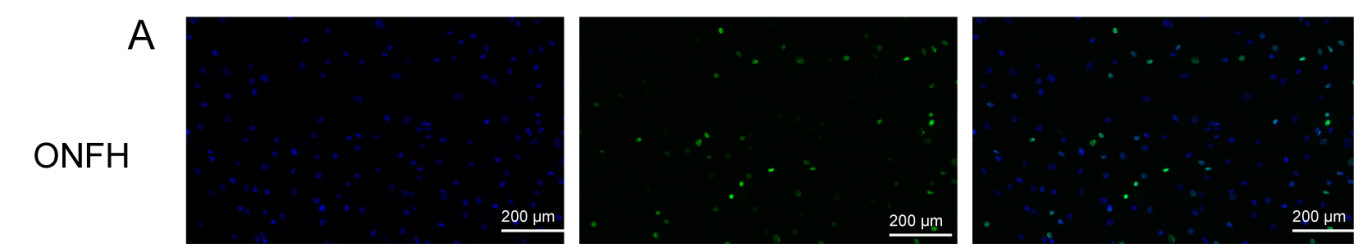

C
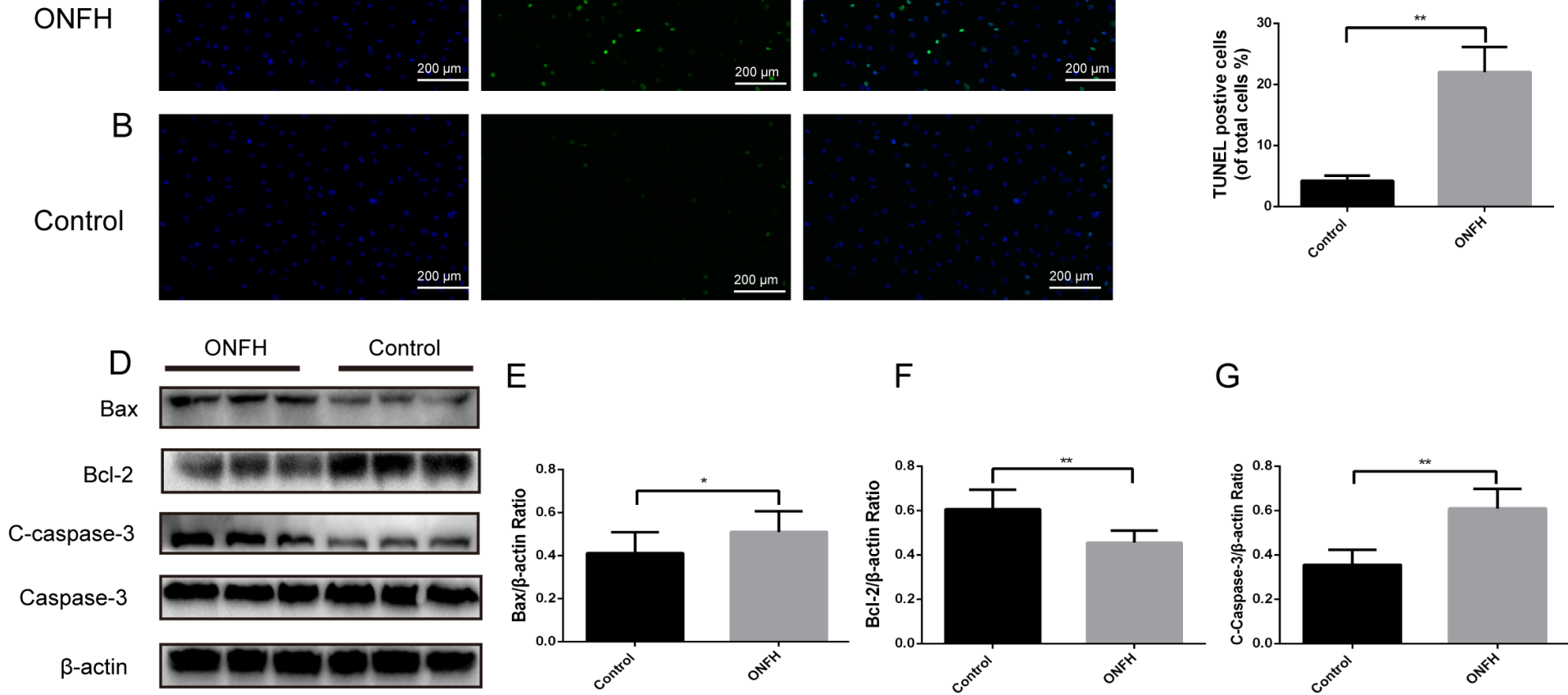

\section{$E$}

$\mathrm{F}$

G
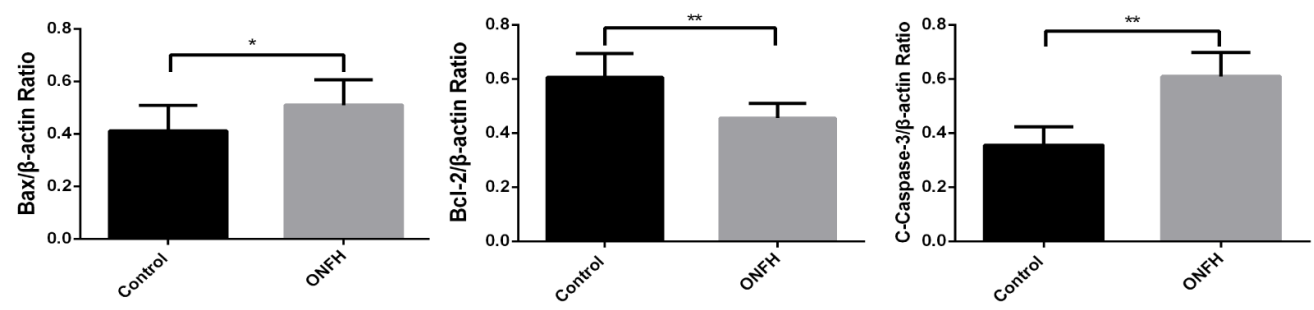

\section{Figure 4}

Apoptotic activity of BMECs. (A-C) Specimens from both groups were subjected to TUNEL assay. Analysis of the results indicated that TUNEL positive cells were markedly higher in ONFH groups in comparison with those in the control group ( $* *$ indicates $P<0.01$ ). Western blot and quantification analyses revealed the protein quantity of caspase-3, Bax, and Bcl-2 in BMECs of both groups. Each assay and analysis was run at least thrice ( $*$ indicates $P<0.05$; ** indicates $P<0.01$ ). 